\begin{tabular}{|c|l|}
\hline Title & Biogenetically inspired synthesis and skeletal diversification of indole al kal oids \\
\hline Author(s) & Mizoguchi, Haruki; Oikawa, Hideaki; Oguri, Hiroki \\
\hline Citation & $\begin{array}{l}\text { Nature chemistry, 6(1), 57-64 } \\
\text { https://doi.org/10.1038NCHEM.1798 }\end{array}$ \\
\hline Issue Date & 201401 \\
\hline Doc URL & http:/hdl.handle.net/2115/57295 \\
\hline Type & article (author version) \\
\hline File Information & oguri.pdf \\
\hline
\end{tabular}

Instructions for use 


\title{
Biogenetically inspired synthesis and skeletal diversification of indole alkaloids
}

\author{
Haruki Mizoguchi $^{1}$, Hideaki Oikawa ${ }^{1}$ and Hiroki Oguri ${ }^{1,2 *}$
}

\author{
${ }^{1}$ Division of Chemistry, Graduate School of Science, Hokkaido University, North 10, West 8, \\ Kita-ku, Sapporo 060-0810, Japan \\ ${ }^{2}$ JST, PRESTO, 4-1-8 Honcho, Kawaguchi, Saitama, 332-0012, Japan
}

E-mail: oguri@sci.hokudai.ac.jp

Abstract: To access architecturally complex natural products, chemists usually devise a customized synthetic strategy for constructing a single target skeleton. In contrast, biosynthetic assembly lines often employ divergent intramolecular cyclizations of a polyunsaturated common intermediate to produce diverse arrays of scaffolds. With the aim of integrating such biogenetic strategies, here we show the development of an artificial divergent assembly line generating unprecedented numbers of scaffold variations of terpenoid indole alkaloids. This approach allows not only practical access to multipotent intermediates but also systematic diversification of skeletal, stereochemical, and functional group properties without structural simplification of naturally occurring alkaloids. Three distinct modes of [4+2] cyclizations and two types of redox-mediated annulations provided divergent access to five skeletally distinct scaffolds involving iboga-, aspidosperma-, andranginine-, and ngouniensine-type skeletons and a non-natural variant within 6-9 steps from tryptamine. The efficiency of our approach was demonstrated by successful total syntheses of $( \pm)$-vincadifformine, $( \pm)$-andranginine, and (-)-catharanthine.

Natural products often bear a variety of functional groups on a rigid, architecturally complex, and $\mathrm{sp}^{3}$-rich skeleton, which allows specific molecular recognition through multi-point interactions to modulate the functions of target biomacromolecules. Chemical synthesis of natural products and their analogs could provide optimum screening collections for the development of drug candidates 
with higher hit rates and a lower probability of side effects. ${ }^{1-3}$ While some innovative synthetic approaches have been recently reported to gain efficient access to such molecules as specific modulators of challenging biological targets, ${ }^{4-11}$ a potentially general strategy for the development of divergent synthetic process to produce assortments of skeletally diverse and densely functionalized scaffolds remains unsettled and needs to be formulated.

In working toward the total synthesis of a natural product composed of complex cyclic arrays, chemists usually develop a customized synthetic approach for the individual targeted scaffold. In contrast, biosynthetic machinery often exploits a common intermediate, conducting divergent transformations to furnish assortments of architecturally distinct skeletons. For instance, a biogenetic hypothesis for monoterpene indole alkaloids is outlined in Fig. $1 .{ }^{12}$ Multistep enzymatic transformations commence with the assembly of tryptamine (1) and secologanin (2) to produce preakuammicine (3). Cleavage of two $\mathrm{C}$-C bonds in the central core of $\mathbf{3}$ generates the hypothetical key intermediate dehydrosecodine (4), composed of a pair of diene units, a dihydropyridine (DHP), and a vinylindole. The intermediate (4) may undergo divergent Diels-Alder-type reactions in either of two ways, with the DHP group serving as a dienophile or diene to form the aspidosperma-type alkaloid tabersonine (5) (path A) or the iboga-type catharanthine (6) (path B). ${ }^{13}$ In addition, a distinct biosynthetic [4+2] cyclization is postulated for andranginine (7) (path C), involving an intermediate bearing a cross-conjugated triene with a higher oxidation level than $4 .^{14}$ The architectural complexity as well as the skeletal variation of the cognate alkaloids could be pre-encoded into the polyunsaturated structure of the achiral intermediate (4), settled at a branching point of the biogenesis. $^{15}$ 


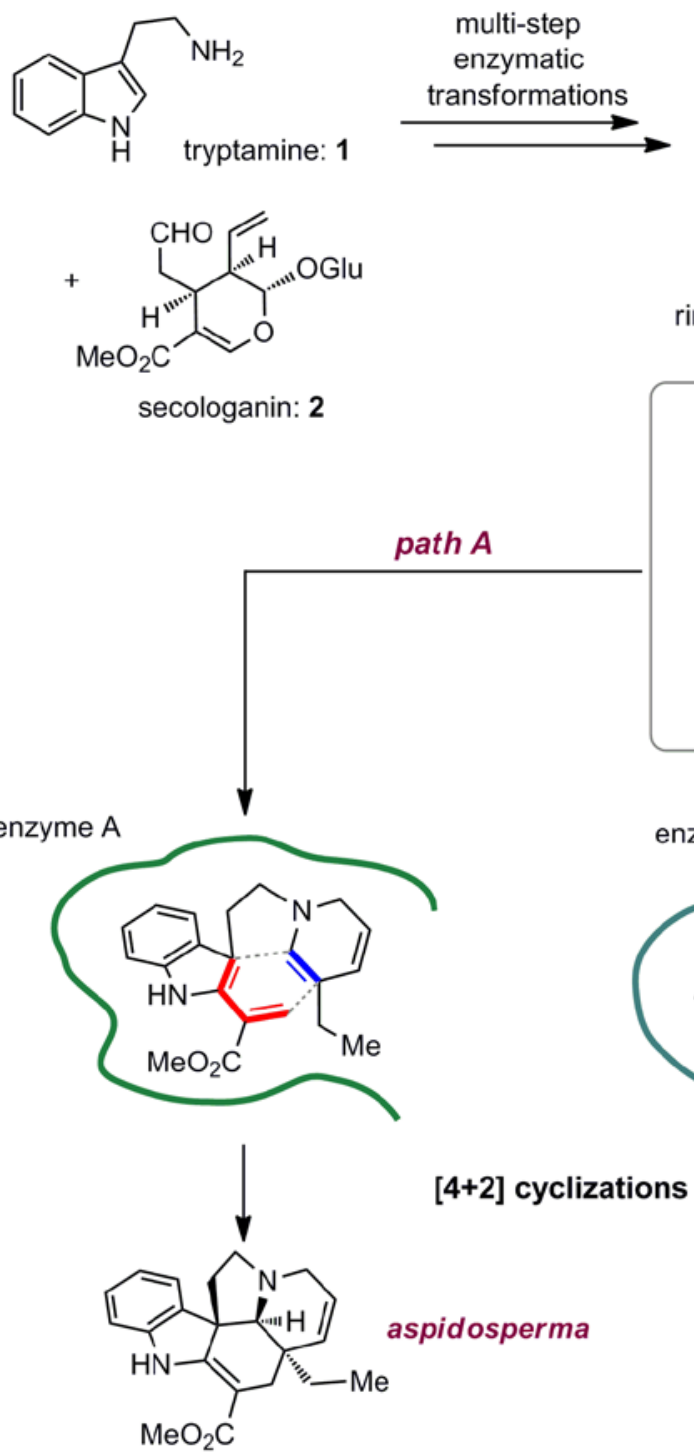

tabersonine: $\mathbf{5}$

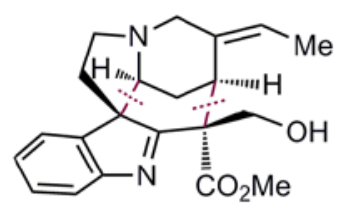

preakuammicine: $\mathbf{3}$

ring-cleavage

hypothetical intermediate

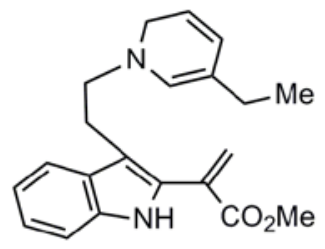

dehydrosecodine: 4
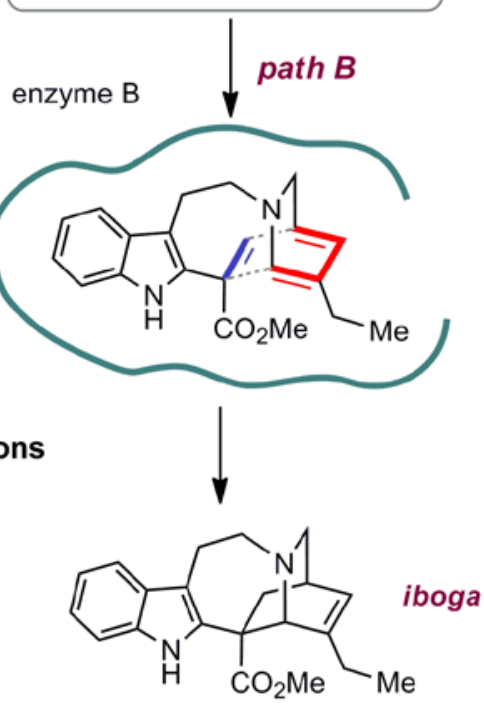

catharanthine: 6

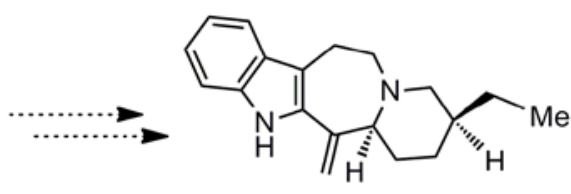

ngouniensine: 8

Figure 1 | Proposed biogenesis of indole alkaloids (5-7) and structure of ngouniensine (8). A hypothetical common intermediate, dehydrosecodine (4) could be biosynthesized through multistep enzymatic transformations starting from union of tryptamine (1) and secologanin (2). A pair of diene units in 4, a dihydropyridine (DHP) and a vinylindole, are thought to be installed through cleavage of two C-C bonds in the central core of an intermediate (3). Divergent [4+2] cyclizations of 4 would form the aspidosperma-type alkaloid tabersonine (5, path A) or the iboga-type catharanthine (6, path B). A distinct biosynthetic [4+2] cyclization is postulated for andranginine (7, path C), involving an intermediate bearing a cross-conjugated triene.

In the biosynthetic assembly line, the protection of the labile intermediates in an enzyme catalytic site provides kinetic stabilization and regulation of multimodal reactivity, allowing cascade synthesis 
with control of chemo-, regio-, stereo-, and even enantioselectivity. In principle, each evolved enzyme possesses a tailored fit that contributes to the catalysis of a particular mode of a skeleton-constructing transformation; ${ }^{16}$ hence, the participation of a series of similar but functionally distinct biomacromolecular catalysts is required for skeletal diversification. Meanwhile, chemical emulation of divergent biogenic processes without the assistance of biomolecular catalysts ${ }^{17}$ pose challenges, including (I) stabilization of the labile intermediates during handling in laboratory, (II) concise and flexible assembly of building blocks, and (III) systematic implementation of various intramolecular cyclization reactions through site-selective activation of polyunsaturated substrates while suppressing competitive reactions. In this study, we aimed to formulate a chemical strategy not only to build a bioinspired assembly line but also to achieve systematic diversification of the skeletal and stereochemical properties of multicyclic scaffolds without structural simplification of the natural products involved. Herein, we report the successful development of a divergent synthetic process, which generates unprecedented levels of skeletal variation of monoterpene indole alkaloids. 
a
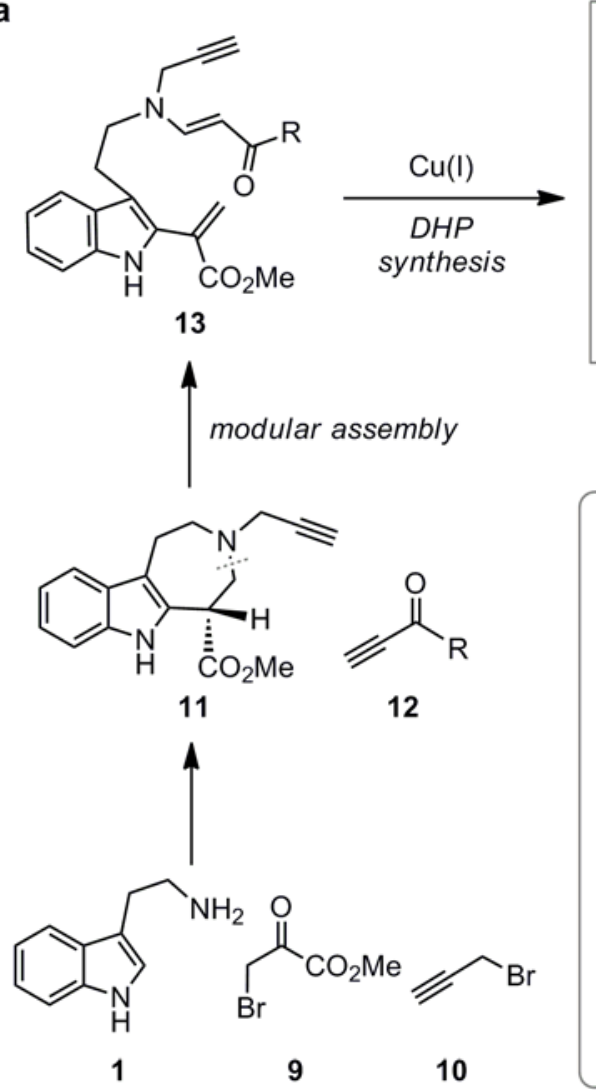

b<smiles>C#CCN(/C=C/C(C)=O)CCc1c[nH]c2ccccc12</smiles>

multipotent intermediate

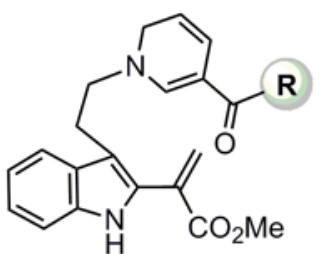

DHP-vinylindole: 14

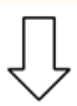

redox-mediated cyclizations

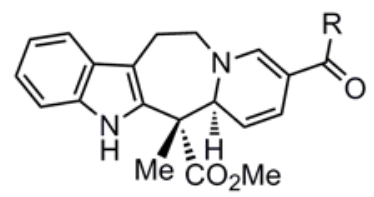

ngouniensine-type: 18

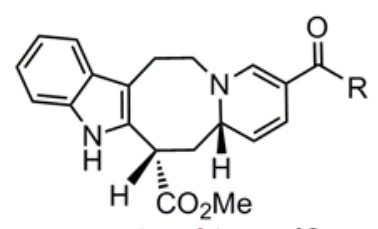

unnatural-type: 19

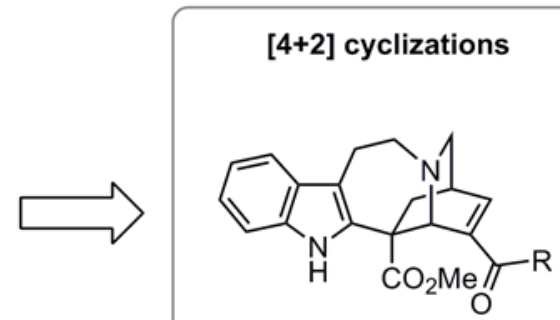

iboga-type: 15

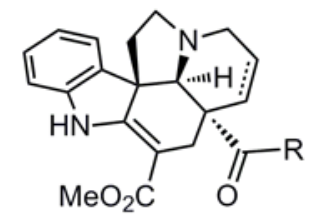

aspidosperma-type: 16

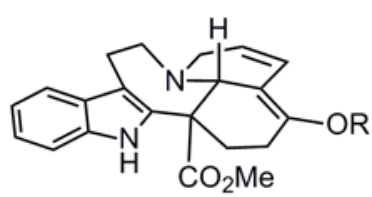

andranginine-type: 17

Figure 2 | Outline of a biogenetically inspired synthetic process to furnish alkaloidal scaffolds. a, Synthetic strategy for a divergent process featuring reactivity modulation of an achiral poly-unsaturated intermediate (14) through manipulation of the carbonyl group conjugated with the dihydropyridine (DHP) unit. Modular assembly of tryptamine (1) and building blocks (9, 10 and 12) followed by cyclization of ene-yne (13) would allow rapid and flexible access to the common intermediate (14). Three distinct modes of bioinspired [4+2] cyclizations could produce assortments of naturally occurring scaffolds (15-17). Distinct modes of annulations were devised through redox-activations of the DHP unit to furnish either the tetracyclic framework (18) of ngouniensine or the unnatural skeleton (19). b, Rapid formation of 1,6-DHP ring using $\mathrm{Cu}(\mathrm{I})$ catalysis. The optimum conditions for the 6-endo cyclization of ene-yne were explored using the model substrate (20). dppf, 1,1'-bis(diphenylphosphino)ferrocene. 


\section{Results and discussion}

Design of the divergent synthetic process. To explore a biogenetically inspired synthetic approach, we first considered the dehydrosecodine (4). Unfortunately, the hypothetical intermediate (4) has not been isolated or synthesized, presumably because the DHP unit of $\mathbf{4}$, containing an ethyl substituent, may be labile to oxidation and oligomerization. In this study, we designed the artificial pluripotent intermediate (14) with intention of stabilizing the polyunsaturated system and thus installed an electron-withdrawing carbonyl group in place of the ethyl substituent (Fig. 2a). In addition, the attachment of various substituents to the carbonyl group could modify the reactivity of the DHP unit for systematic implementation of intramolecular cyclizations with control of regio- and stereoselectivity. We devised three types of bioinspired [4+2] cyclizations of $\mathbf{1 4}$, leading to $\mathbf{1 5 - 1 7}$, by manipulating the DHP unit conjugated with the carbonyl group. Moreover, redox activations of the DHP unit of $\mathbf{1 4}$ were explored to achieve distinct modes of annulations to furnish either the tetracyclic framework (18) of ngouniensine $(\mathbf{8})^{18}$ or the unnatural skeleton (19). We also conceived a late-stage chiral induction by attaching an auxiliary to the carbonyl group to accomplish enantioselective synthesis.

The sensitive DHP unit in the intermediate (14) should be formed just prior to the divergent cyclization reactions. Retrosynthetically, chemoselective activation of the alkynyl group in ene-yne (13) would allow 6-endo cyclization to afford $\mathbf{1 4}$. With the intention of flexibly incorporating a series of enaminocarbonyl moieties into the precursors, we devised a union of a tricyclic precursor (11) with an ethynylcarbonyl compound (12), accompanied by Hofmann elimination, to generate the ene-yne (13) containing a vinylindole unit. The precursor (11), a $\beta$-amino acid derivative, should be readily available by assembly of tryptamine (1) with methyl 3-bromo-2-oxopropanoate (9) and propargyl bromide (10). 
Development of Cu(I)-catalyzed cyclization to form the DHP ring. With this strategic planning in mind, we first sought to develop an expedient protocol for the conversion of ene-yne (13) to the DHP-vinylindole intermediate (14) (Fig. 2b). Whereas approaches to the synthesis of substituted pyridines via oxidation of temporarily formed DHPs have been relatively well explored, ${ }^{19-21}$ protocols for obtaining DHP rings via metal-catalyzed 6-endo cyclization of $N$-propargyl enaminocarbonyls are limited. $^{22,23}$ In our system, rapid construction of the 1,6-DHP ring was required under very mild conditions without causing further intra- and intermolecular reactions of the elaborate $\pi$-conjugated systems in $\mathbf{1 4}$. As shown in Fig. $2 \mathrm{~b}$, investigation using the model substrate (20) allowed us to identify the optimum conditions that employ a cationic $\mathrm{Cu}(\mathrm{I})$ catalyst as an efficient alkyne activator. Upon treatment of $\mathbf{2 0}$ with a catalytic amount (10 mol\%) of $[\mathrm{Cu}(\mathrm{dppf})(\mathrm{MeCN})] \mathrm{PF}_{6}{ }^{24}$ in dichloromethane, 6-endo cyclization proceeded smoothly within 60 min at room temperature to afford the desired 1,6-DHP (21) in 97\% yield without affecting the non-protected indole or the resulting DHP ring located in the vicinity. 


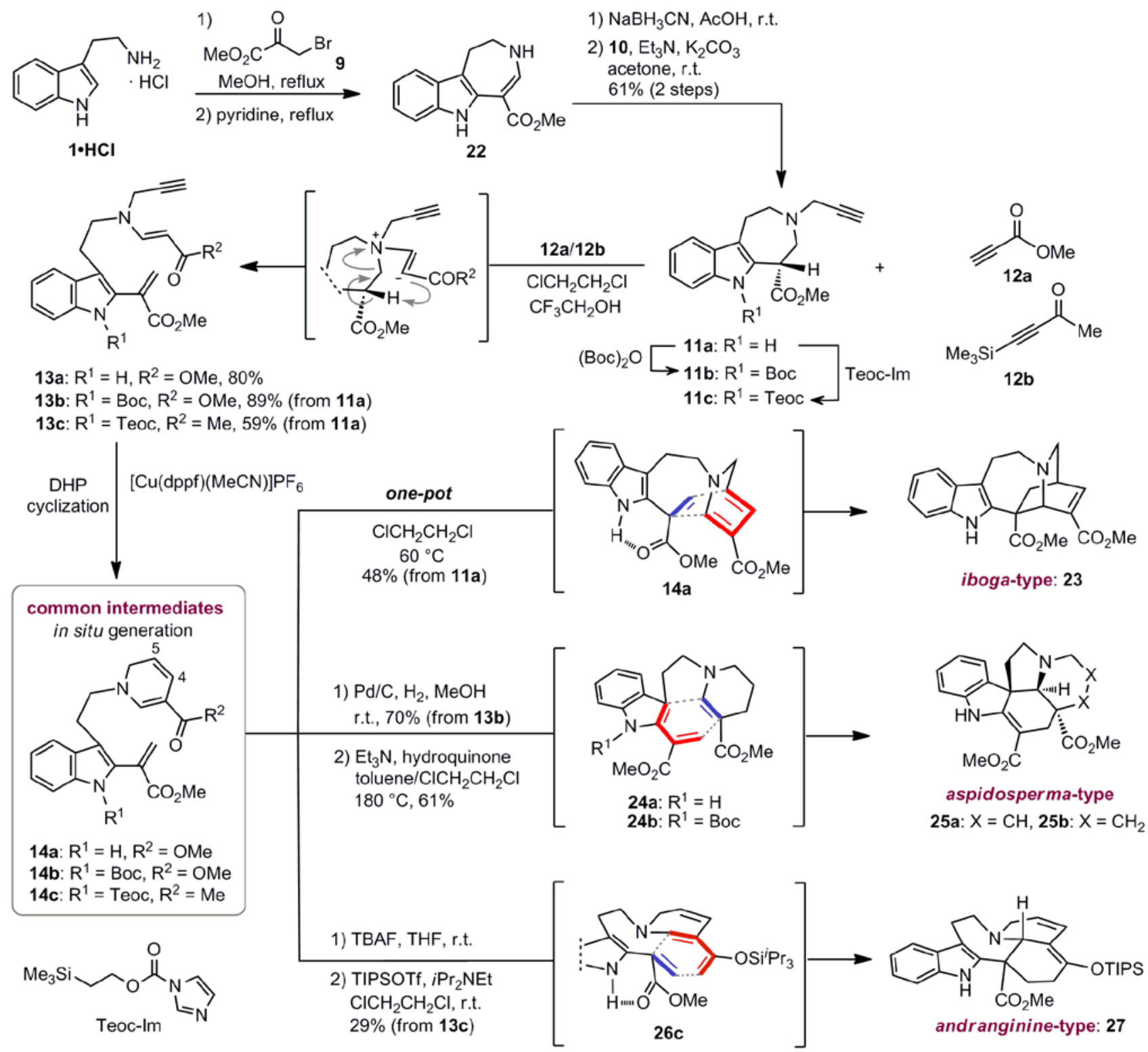

Figure 3 | Unified synthetic process for divergent access to 23, 25, and 27 through distinct [4+2] cyclization reactions. Pictet-Spengler reaction of tryptamine (1) with $\mathbf{9}$ and subsequent ring expansion produced 22. Reduction followed by $N$-propargylation gave 11a. Assemblies of 11 with ethynylcarbonyl unit (12) could generate a zwitterionic intermediate effecting Hofmann elimination to afford ene-yne (13). Cu(I)-catalyzed cyclization of 13a followed by [4+2] cyclization allowed a streamlined synthesis of iboga-type scaffold 23. The aspidosperma-type scaffold (25b) was synthesized through regioselective hydrogenation of $\mathbf{1 4 b}$ and a different [4+2] cyclization. A distinct [4+2] cyclization was realized through conversion of 14c into 26c to produce andranginine-type scaffold (27). Boc, t-butoxycarbonyl; Teoc, 2-(trimethylsilyl)ethoxycarbonyl; Im, imidazole; dppf, 1,1'-bis(diphenylphosphino)ferrocene; DHP, dihydropyridine; TBAF, tetra-n-butylammonium fluoride; TIPSOTf, triisopropylsilyl trifluoromethanesulfonate. 
Synthesis of a precursor for DHP cyclization, ene-yne (13a). Having developed a sensible approach to formation of the 1,6-DHP ring, we synthesized ene-yne (13a), bearing a vinylindole unit, from tryptamine (1) in 5 steps (Fig. 3). A Pictet-Spengler reaction of $\mathbf{1}$ with $\mathbf{9}$ and subsequent ring expansion gave 22, based on a reported protocol. ${ }^{25}$ Reduction of 22 with sodium cyanoborohydride in acetic acid and subsequent $N$-propargylation produced 11a in 61\% yield (2 steps). Simultaneous installation of the enaminoester group and the gem-substituted vinylindole unit was efficiently achieved upon treatment of 11a with methyl propiolate (12a) to furnish 13a. Presumably, a zwitterionic intermediate would be capable of causing regioselective Hofmann elimination spontaneously. The use of 2,2,2-trifluoroethanol as a co-solvent with 1,2-dichloroethane was critical in gaining single-step access to $\mathbf{1 3 a}$ in greater than $80 \%$ yield. Since the ene-yne (13a) was prone to gradual dimerization under concentrated conditions, it was used directly in subsequent conversions without chromatographic purification in most cases.

Bioinspired divergent [4+2] cyclization reactions. Next, the formation of DHP-vinylindole intermediate (14) and the implementation of a series of [4+2] cyclization reactions were explored (Fig. 3). Upon treatment of a crude mixture containing 13a with $[\mathrm{Cu}(\mathrm{dppf})(\mathrm{MeCN})] \mathrm{PF}_{6}(10 \mathrm{~mol} \%)$ at $60^{\circ} \mathrm{C}$, a cascade of reactions-DHP formation $(\mathbf{1 3 a} \rightarrow \mathbf{1 4 a})$ and subsequent Diels-Alder-type reaction-proceeded smoothly in one-pot to produce iboga-type (23) in 48\% yield from 11a. The streamlined synthesis of the iboga skeleton possessing an additional carbonyl functional group was achieved in 6 steps from 1 without protection of the indole group. In contrast, for substrates bearing a tert-butoxylcarbonyl (Boc) group or a simple methyl substituent at the indole N1 position, the same conversion produced only trace amounts of the corresponding iboga-type cycloadducts, whereas $\mathrm{Cu}(\mathrm{I})$-catalyzed DHP formation took place efficiently regardless of the indole substituent (see Supporting Information). It is likely that the contribution of hydrogen bonding between the indole $\mathrm{NH}$ and the carbonyl oxygen of the $\alpha, \beta$-unsaturated methylester in 14a not only increased the 
electrophilicity of the dienophile but also fixed the conformation appropriately for cyclization to form the iboga-skeleton (23).

Next, we aimed to achieve a distinct mode of [4+2] cyclization leading to the aspidosperma-skeleton (25) (Fig. 3). Despite extensive efforts to activate 14a with various Lewis/Brønsted acids and bases, formation of the iboga-skeleton (23) and oxidation to pyridinium salts occurred predominantly. The aspidosperma-skeleton (25a) was obtained, in less than 5\% yield, only when 14a was carefully treated with tert-butyldimethylsilyl trifluoromethanesulfonate (TBSOTf) at low temperature (see Supporting Information). In order to suppress the formation of the iboga-skeleton as well as the oxidation of the DHP ring, we were obliged to protect the indole nitrogen and also to reduce the C4-C5 double bond in the DHP ring. Protection of 11a with a Boc group and subsequent assembly of $\mathbf{1 1 b}$ with 12a via Hofmann elimination gave ene-yne (13b) in good yields. $\mathrm{Cu}(\mathrm{I})$-catalyzed DHP formation and subsequent site-selective hydrogenation of the resulting 14b provided isolable tetrahydropyridine (24b) in 70\% yield (2 steps). Microwave-assisted heating of $\mathbf{2 4 \mathbf { b }}$ at $180{ }^{\circ} \mathrm{C}$ with hydroquinone (a polymerization inhibitor) and triethylamine ${ }^{26}$ effected thermal decomposition of the Boc group (24b $\rightarrow \mathbf{2 4 a}$ ) followed by [4+2] cyclization, giving rise to the aspidosperma-skeleton (25b) in 61\% yield.

We then turned our attention to achieving a distinct [4+2] annulation via intermediate (26c) bearing cross-conjugated triene to yield the andranginine-type scaffold (27) (Fig. 3). To this end, we examined the installation of a methylketone group into the versatile intermediate (14c) to generate the triene (26c) through silylenol ether formation. $\mathrm{Cu}(\mathrm{I})$-catalyzed 6-endo cyclization of the corresponding ene-yne (13c) bearing the methylketone group, however, required gentle heating at $45^{\circ} \mathrm{C}$ to form the DHP ring. From a practical point of view, protection of the indole nitrogen should be beneficial in suppressing competitive cyclization leading to the iboga-skeleton and other side reactions. In fact, protection with a 2-(trimethylsilyl)ethoxycarbonyl (Teoc) group (11a $\rightarrow \mathbf{1 1 c}$ ) 
ensured stabilities of $13 \mathrm{c}$ and $\mathbf{1 4 c}$ upon conversion of 11c into 14c. The tricycle (11c) and 4-trimethylsilyl-3-butyne-2-one (12b) were efficiently assembled via Hofmann elimination to give the desired ene-yne (13c) with the loss of a trimethylsilyl group. The resulting 13c was subjected to sequential operations- $\mathrm{Cu}(\mathrm{I})$-catalyzed DHP formation at $45^{\circ} \mathrm{C}$, producing $14 \mathrm{c}$, and removal of the Teoc group with tetrabutylammonium fluoride (TBAF) followed by treatment with triisopropylsilyl trifluoromethanesulfonate (TIPSOTf) at room temperature- to generate the intermediate (26c) having a silylenol ether. The desired mode of [4+2] cyclization of 26c occurred spontaneously to furnish the andranginine-type scaffold (27) (29\% for 3 steps from 13c). Thus far, we have implemented three distinct bioinspired Diels-Alder-type cyclization reactions of intermediates (14a, 24a, and 26c) leading to a series of alkaloidal scaffolds, 23, 25b, and 27.

Redox-mediated cyclization reactions leading to 28 and 29. Further skeletal diversifications were then explored through redox activation of the DHP unit (Fig. 4). To achieve an alternative cyclization of 14a, we first envisaged two-electron oxidation of the DHP ring to generate a pyridinium species. Indeed, when the reaction mixture for the conversion of $\mathbf{1 3 a}$ to $14 \mathbf{a}$ was heated to $120^{\circ} \mathrm{C}$, a distinct annulation of $\mathbf{1 4 a}$ took place predominantly, giving tetracyclic scaffold (28) in good yield (57\% from 11a). The tetracyclic array of $\mathbf{2 8}$ is identical to the framework of naturally occurring ngouniensine (8). ${ }^{27}$ The relative configuration of $\mathbf{2 8}$ was elucidated based on X-ray analysis of the crystalline equivalents (see Supporting Information). Taking into account for the experimental results on the conversions of deuterium labeled 14a (see Supplementary Schemes S1-S2), $\mathbf{2 8}$ could presumably be formed through a hydride shift generating a zwitterionic intermediate composed of a pyridinium cation and an enolate anion, which would undergo subsequent 7 -endo cyclization. ${ }^{28}$ In contrast to the identical conversion of 13a into 14a under gentle heating at $60^{\circ} \mathrm{C}$, yielding the iboga-skeleton (23) as the major product (Fig. 3), 23 was not obtained at all at $120^{\circ} \mathrm{C}$. We thus postulated the involvement of a retro-Diels-Alder reaction. When isolated 23 was heated to $120^{\circ} \mathrm{C}$, as expected, we 
observed the consumption of $\mathbf{2 3}$ and the formation of $\mathbf{2 8}$ as the major product (47\%, see Supporting Information). It seems probable that the retro-Diels-Alder reaction of kinetically formed 23 regenerates the DHP-vinylindole intermediate (14a) at the elevated temperature. Hydride transfer and subsequent cyclization may occur irreversibly to furnish ngouniensine-type scaffold (28).

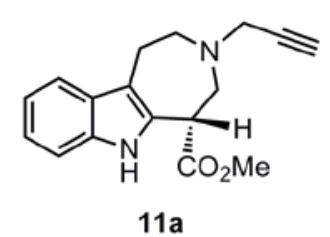

$11 \mathrm{a}$
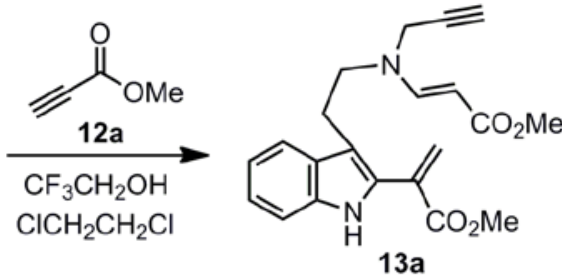

Cu(dppf)(MeCN)]PF 6
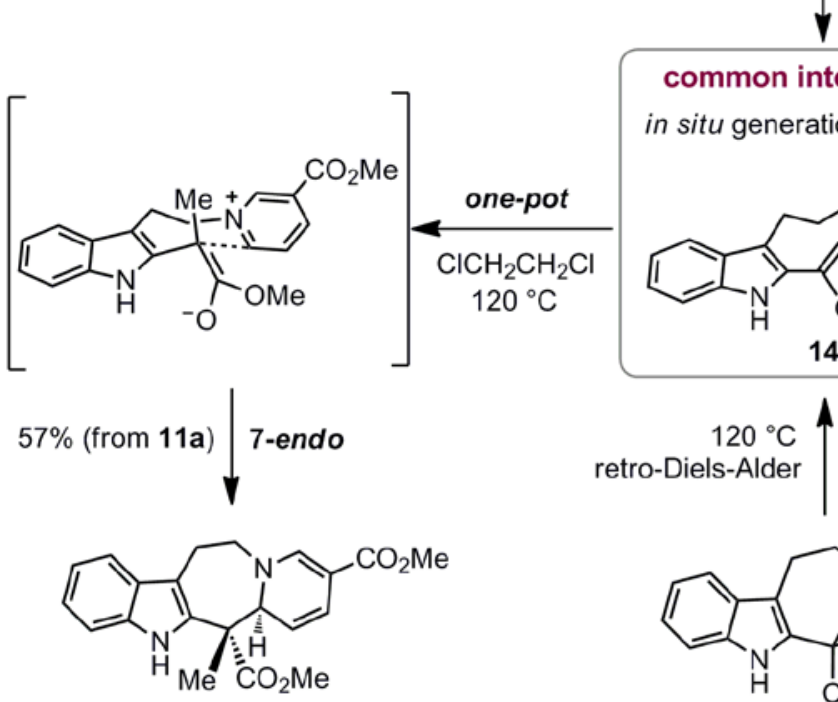

ngouniensine-type: 28

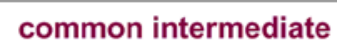

in situ generation $\quad \mathrm{CO}_{2} \mathrm{Me}$

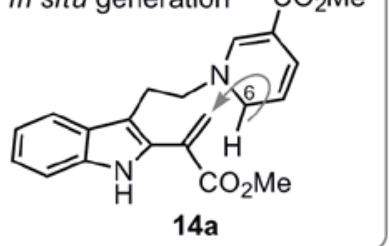

$14 a$
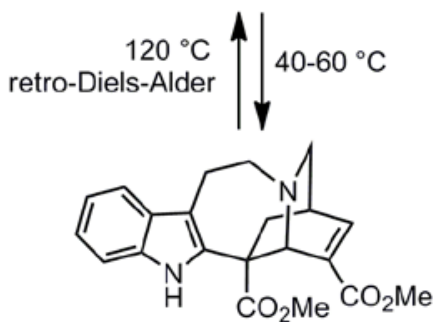

iboga-type: 23
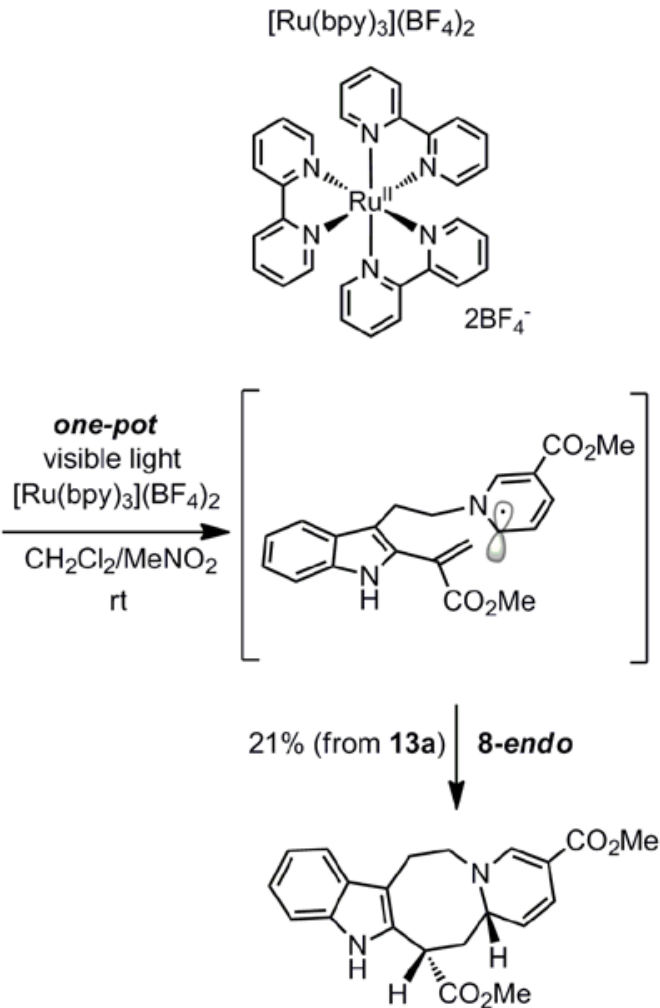

unnatural-type: 29

Figure 4 | Redox-mediated activation of the DHP-vinylindole (14a) leading to tetracyclic scaffolds (28 and 29), which contain a DHP ring. The common intermediate (14a) generated from 11a was subjected to either two- or single-electron oxidation process to produce $\mathbf{2 8}$ and $\mathbf{2 9}$, respectively. Upon heating at $120^{\circ} \mathrm{C}$, ngouniensine-type scaffold (28) was obtained as the major product. The involvements of the retro-Diels-Alder reaction $(\mathbf{2 3} \rightarrow \mathbf{1 4 a})$, hydride shift followed by 7-endo-cyclization of a zwitterionic intermediate were postulated in the formation of $\mathbf{2 8 .}$ Single-electron oxidation of 14a provided an unnatural-type scaffold (29) presumably via 8-endo-radical cyclization. bpy, 2,2'-bipyridine; dppf, 1,1'-bis(diphenylphosphino)ferrocene.

Next, we envisioned single-electron oxidation of the 1,6-DHP ring in 14a to generate a carbon-centered radical species. Since photo-redox synthetic processes often employ 
1-benzyl-1,4-dihydronicotinamide (BNAH) with a 1,4-DHP system as a versatile reductant, ${ }^{29-31} \mathbf{1 4 a}$ was treated with a photo-redox catalyst, $\left[\mathrm{Ru}(\mathrm{bpy})_{3}\right]\left(\mathrm{BF}_{4}\right)_{2}$, at $0{ }^{\circ} \mathrm{C}$ under irradiation with fluorescent light (12 W). The tetracyclic scaffold (29), not found in naturally occurring alkaloids, was obtained, albeit in low yield (21\%), which may have been consistent with our intention to conduct a redox-mediated 8-endo cyclization. Oxidative activations of 14a in either a two-electron or a single-electron process produced the tetracyclic scaffolds (28 and 29) bearing DHP ring in their fused scaffolds, which could provide a versatile platform for further diversification. In this way, we illustrated the systematic generation of skeletally diverse scaffolds (23, 25, and 27-29) relevant to naturally occurring indole alkaloids within 6-9 steps from tryptamine (1).

Total synthesis of three natural products. To highlight the applicability and efficiency of the divergent synthetic process, we sought to achieve total syntheses of ( \pm )-vincadifformine (32), ( \pm )-andranginine (7), and (-)-catharanthine (6) (Fig. 5). Since the aspidosperma-type scaffold (25b) bears the requisite functional array of ( \pm )-vincadifformine (32) (Fig. 5a), we carried out a total synthesis to confirm its structure (see Supporting Information for precedent total syntheses of 32). ${ }^{5}$ 32-35 The more sterically hindered methyl ester in $\mathbf{2 5} \mathbf{b}$ was reduced site-selectively by simple treatment with diisobutylaluminium hydride (DIBAL) to furnish primary alcohol $\mathbf{3 0}$ in $87 \%$ yield. Oxidation of $\mathbf{3 0}$ and subsequent Wittig elongation produced 31, which contains a vinyl group. Site-selective hydrogenation of 31 gave rise to ( \pm )-vincadifformine (32) in $77 \%$ yield. 
a

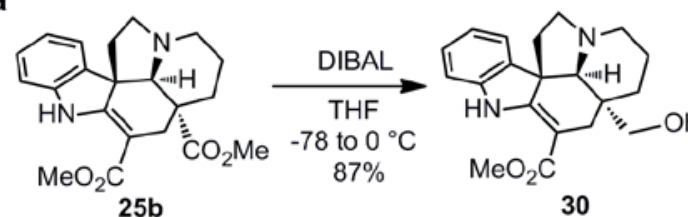

30

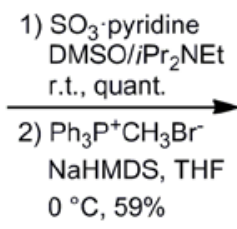

$0{ }^{\circ} \mathrm{C}, 59 \%$

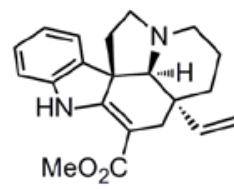

31
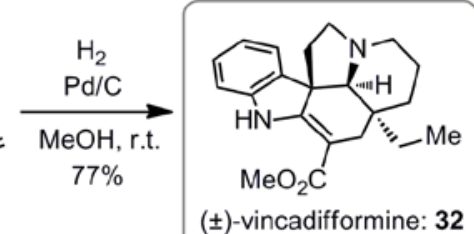

b

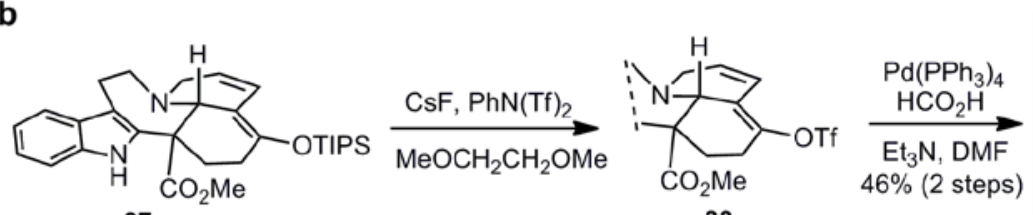

27

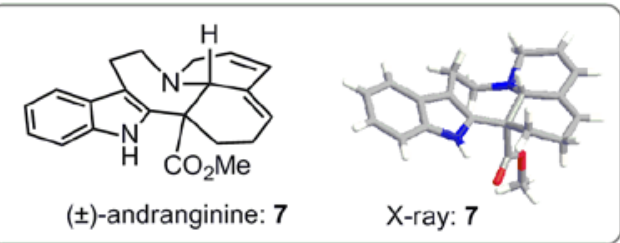

c<smiles>C#CCN1CCc2c([nH]c3ccccc23)[C@]([CH2+])(C(C)=O)CN1CC#CC(=O)N1C(=O)OCC1C(C)C</smiles>

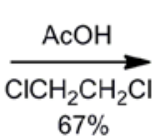<smiles>[M]C(C)C1COC(=O)N1C(=O)/C=C/N(CC#C)CCc1c(C(=O)OC)[nH]c2ccccc12</smiles>

34

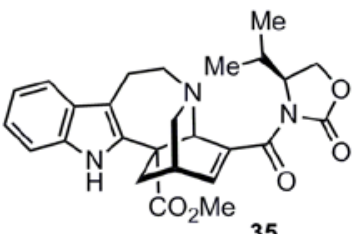

35
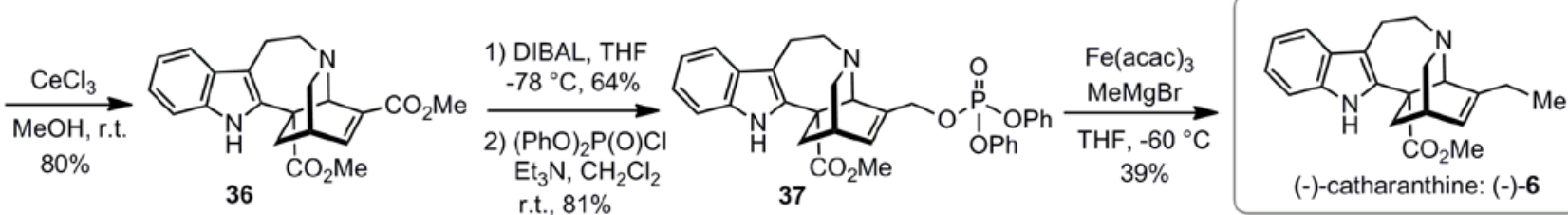

Figure 5 | Total synthesis of three natural products. a, Site-selective reduction of methylester (25b) followed by oxidation of resulting primary alcohol and Wittig elongation gave $\mathbf{3 1}$.

Site-selective hydrogenation of $\mathbf{3 1}$ afforded ( \pm )-vincadifformine (32). b. Direct conversion of silylenol ether (27) to vinyl triflate (33) and subsequent palladium-catalyzed reduction furnished ( \pm )-andranginine (7). c, Hofmann elimination triggered by addition of 11a to 12c gave 34 . After $\mathrm{Cu}(\mathrm{I})$-catalyzed formation of DHP ring, the critical [4+2] cyclization reaction proceeded with complete diastereocontrol in one-pot to produce 35. Removal of the chiral auxiliary, site-selective reduction of 36, and conversion into an allylic phosphate (37) followed by Fe-catalyzed cross-coupling with MeMgBr accomplished asymmetric total synthesis of (-)-catharanthine (6). DIBAL, diisobutylaluminium hydride; NaHMDS, sodium hexamethyldisilazide; Tf, trifluoromethanesulfonyl; dppf, 1,1'-bis(diphenylphosphino)ferrocene; acac, acetylacetonate.

Next, the conversion of pentacycle (27) to ( \pm )-andranginine (7) was accomplished by trimming an additional functional group in two steps (Fig. 5b). Direct conversion of $\mathbf{2 7}$ bearing a silylenol ether to vinyl triflate (33) ${ }^{36}$ and subsequent palladium-catalyzed reduction furnished $\mathbf{7}$ in good yield. X-ray analysis of crystalline 7 confirmed its relative configuration, which was identical to the proposed 
structure of andranginine. ${ }^{37}$ This is the first example of de novo total synthesis of the almost disregarded natural product, $\left( \pm\right.$ )-andranginine (7), in 11 steps, ${ }^{38}$ which lends experimental support to an alternate mode of biosynthetic Diels-Alder-type cyclization diverging from the hypothetical intermediate (4).

We then performed enantioselective total synthesis of catharanthine (6) (Fig. 5c). To illustrate late-stage asymmetric induction through attachment of a chiral auxiliary to the DHP ring, ene-yne (34) with an oxazolidinone group was synthesized by exploiting the modular nature of the assembly line. Hofmann elimination triggered by addition of 11a to 12c proceeded efficiently under modified conditions to produce 34 in 67\% yield with the loss of a stereogenic center in racemic tricycle (11a). Upon treatment of 34 with the $\mathrm{Cu}(\mathrm{I})$ catalyst (15 mol\%) at $45{ }^{\circ} \mathrm{C}$, a cascade transformation afforded the desired iboga-scaffold (35) in 48\% yield with complete diastereocontrol of the critical [4+2] cyclization reaction. Removal of the chiral auxiliary by $\mathrm{CeCl}_{3}$-catalyzed methanolysis gave the corresponding methyl ester (36) in 80\% yield. Site-selective reduction of 36 with DIBAL produced an allylic alcohol, which was then transformed to an allylic phosphate (37) in good yield. An iron-catalyzed cross-coupling reaction with methyl Grignard reagent ${ }^{39}$ proceeded regioselectively accomplishing asymmetric total synthesis of (-)-catharanthine (6). The absolute configuration was assigned based on optical rotation (see Supporting Information). While there has been pioneering synthetic studies, ${ }^{26,40-49}$ the 10-step sequence described herein is the shortest enantiocontrolled total synthesis of (-)-catharanthine (6), to the best of our knowledge.$^{50}$ Consequently, we have successfully demonstrated that the appropriately functionalized scaffolds generated via three distinct modes of the bioinspired [4+2] cyclizations are readily applicable for achieving total syntheses of ( \pm )-vincadifformine (32), ( \pm )-andranginine (7), and (-)-catharanthine (6).

In summary, we have developed a unified synthetic process generating unprecedented levels of scaffold variations of natural products without structural simplification. The multipotent DHP-vinylindole intermediates (14a-c) were flexibly synthesized through unions of tricycles 
(11a-c) and ethynylcarbonyl units (12a-c) followed by $\mathrm{Cu}(\mathrm{I})$-catalyzed formation of the DHP ring. By harnessing the versatile reactivity of $\mathbf{1 4}$, multiple modes of annulation were systematically implemented. The divergent process allowed a concise and programmable access to four naturally occurring scaffolds (23, 25b, 27, and 28) and a non-natural skeletal variant (29), each within 6-9 steps from tryptamine (1). This synthetic campaign illustrates the concept of reactivity modulation of an achiral poly-unsaturated intermediate in conjunction with modular assembly of building blocks and regio/stereo-controlled cyclizations, which form a foundation for the development of a divergent synthetic process generating a series of natural products and their structural variants having different skeletal, stereochemical and functional group properties. The synthetic strategies and tactics demonstrated herein could be applicable to design artificial assembly lines to furnish collections of natural product-inspired small molecules by emulating biogenesis of other families of secondary metabolites.

\section{References}

1. Ganesan, A. The impact of natural products upon modern drug discovery. Curr. Opin. Chem. Biol. 12, 306-317 (2008).

2 Nielsen, T. E. \& Schreiber, S. L. Towards the optimal screening collection. A synthesis strategy. Angew. Chem., Int. Ed. 47, 48-56 (2008).

3. Lachance, H., Wetzel, S., Kumar, K. \& Waldmann, H. Charting, Navigating, and Populating Natural Product Chemical Space for Drug Discovery. J. Med. Chem. 55, 5989-6001 (2012).

4. Young, I. S. \& Baran, P. S. Protecting-group-free synthesis as an opportunity for invention. Nature Chem. 1, 193-205 (2009).

5. Jones, S. B., Simmons, B., Mastracchio, A. \& MacMillan, D. W. C. Collective synthesis of natural products by means of organocascade catalysis. Nature 475, 183-188 (2011).

6. Snyder, S. A., Gollner, A. \& Chiriac, M. I. Regioselective reactions for programmable resveratrol oligomer synthesis. Nature 474, 461-465 (2011).

7. Oguri, H. et al. Generation of anti-trypanosomal agents through concise synthesis and structural diversification of sesquiterpene analogs. J. Am. Chem. Soc. 133, 7096-7105 (2011).

8. Balthaser, B. R., Maloney, M. C., Beeler, A. B., Porco, J. A. \& Snyder, J. K. Remodelling of the natural product fumagillol employing a reaction discovery approach. Nature Chem. 3, 969-973 (2011). 
9. Aquino, C. et al. A biomimetic polyketide-inspired approach to small-molecule ligand discovery. Nature Chem. 4, 99-104 (2012).

10. Bauer, R. A., Wenderski, T. A. \& Tan, D. S. Biomimetic diversity-oriented synthesis of benzannulated medium rings via ring expansion. Nature Chem. Biol. 9, 21-29 (2013).

11. Huigens III, R.W. et al. A ring-distortion strategy to construct stereochemically complex and structurally diverse compounds from natural products. Nature Chem. 5, 195-202 (2013).

12. O'Connor, S. E. \& Maresh, J. J. Chemistry and biology of monoterpene indole alkaloid biosynthesis. Nat. Prod. Rep. 23, 532-547 (2006).

13. Stocking, E. M. \& Williams, R. M. Chemistry and biology of biosynthetic Diels-Alder reactions. Angew. Chem. Int. Ed. 42, 3078-3115 (2003).

14. Danieli, B., Lesma, G., Martinelli, M., Passarella, D. \& Silvani, A. Diastereoselective Synthesis of 3-Oxo-14,15-dihydroandranginine. J. Org. Chem. 62, 6519-6523 (1997).

15. Finefield, J. M., Sherman, D. H., Kreitman, M. \& Williams, R. M. Enantiomeric Natural Products: Occurrence and Biogenesis. Angew. Chem. Int. Ed. 51, 4802-4836 (2012).

16. Oikawa, H. \& Tokiwano, T. Enzymatic catalysis of the Diels-Alder reaction in the biosynthesis of natural products. Nat. Prod. Rep. 21, 321-352 (2004).

17. Kim, J. \& Movassaghi, M. Biogenetically inspired syntheses of alkaloid natural products. Chem. Soc. Rev. 38, 3035-3050 (2009).

18. Massiot, G. et al. Structures of ngouniensine: an indole alkaloid of a new type from Strychnos ngouniensis. J. Chem. Soc., Chem. Commun. 768-769 (1982).

19. Abbiati, G. et al. Sequential Amination/Annulation/Aromatization Reaction of Carbonyl Compounds and Propargylamine: A New One-Pot Approach to Functionalized Pyridines. $J$. Org. Chem. 68, 6959-6966 (2003).

20. Cacchi, S., Fabrizi, G. \& Filisti, E. $N$-Propargylic $\beta$-Enaminones: Common Intermediates for the Synthesis of Polysubstituted Pyrroles and Pyridines. Org. Lett. 10, 2629-2632 (2008).

21. Colby, D. A., Bergman, R. G. \& Ellman, J. A. Synthesis of Dihydropyridines and Pyridines from Imines and Alkynes via C-H Activation. J. Am. Chem. Soc. 130, 3645-3651 (2008).

22. Kim, H. \& Lee, C. Rhodium-Catalyzed Cycloisomerization of $N$-Propargyl Enamine Derivatives. J. Am. Chem. Soc. 128, 6336-6337 (2006).

23. Martins, M. A. P. et al. Intramolecular cyclization of $N$-propargylic $\beta$-enaminones catalyzed by silver. Tetrahedron Lett. 54, 847-849 (2013).

24. Kim, H.-S., Kim, J.-W., Kwon, S.-C., Shim, S.-C. \& Kim, T.-J. Catalytic formation of carbamates and cyclic carbonates by copper complex of 2,5,19,22-tetraaza[6,6](1,1')ferrocenophane-1,5-diene X-ray crystal structure of [Cu(I)]PF . $J$. Organomet. Chem. 545-546, 337-344 (1997).

25. Chen, P., Cao, L. \& Li, C. Protecting-Group-Free Total Synthesis of ( \pm )-Subincanadine F. J. Org. Chem. 74, 7533-7535 (2009).

26. Kuehne, M. E., Bornmann, W. G., Earley, W. G. \& Marko, I. Studies in biomimetic alkaloid 
syntheses. 14. Controlled, selective syntheses of catharanthine and tabersonine, and related desethyl compounds, through generation of 15-oxosecodine intermediates. J. Org. Chem. 51, 2913-2927 (1986).

27. Passarella, D., Martinelli, M., Llor, N., Amat, M. \& Bosch, J. Biomimetic construction of the tetracyclic ring system of ngouniensine. Tetrahedron 55, 14995-15000 (1999).

28. Wilson, R. M., Farr, R. A. \& Burlett, D. J. Synthesis and chemistry of a stabilized dehydrosecodine model system. J. Org. Chem. 46, 3293-3302 (1981).

29. Yoon, T. P., Ischay, M. A. \& Du, J. Visible light photocatalysis as a greener approach to photochemical synthesis. Nature Chem. 2, 527-532 (2010).

30. Tucker, J. W. \& Stephenson, C. R. J. Shining Light on Photoredox Catalysis: Theory and Synthetic Applications. J. Org. Chem. 77, 1617-1622 (2012).

31. Prier, C. K., Rankic, D. A. \& MacMillan, D. W. C. Visible Light Photoredox Catalysis with Transition Metal Complexes: Applications in Organic Synthesis. Chem. Rev. 113, 5322-5363 (2013).

32. Kutney, J. P. et al. The total synthesis of some monomeric Vinca alkaloids: $d l$-vincadine, $d l$-vincaminoreine, $d l$-vincaminorine, $d l$-vincadifformine, $d l$-minovine, and dl-vincaminoridine. J. Am. Chem. Soc. 90, 3891-3893 (1968).

33. Kuehne, M. E., Wang, T. \& Seaton, P. J. Total Syntheses of Vincadifformine, 3-Oxovincadifformine, pseudo-and-20-epi-pseudo-Vincadifformine, Tabersonine, and $\Delta$ 18-Tabersonine through Radical Reactions and Heck Reactions. J. Org. Chem. 61, 6001-6008 (1996).

34. Kobayashi, S., Peng, G. \& Fukuyama, T. Efficient total syntheses of ( \pm )-vincadifformine and (-)-tabersonine. Tetrahedron Lett. 40, 1519-1522 (1999).

35. Pandey, G. \& Kumara, C. P. Iminium Ion Cascade Reaction in the Total Synthesis of (+)-Vincadifformine. Org. Lett. 13, 4672-4675 (2011).

36. Mi, Y., Schreiber, J. V. \& Corey, E. J. Total Synthesis of (+)- $\alpha$-Onocerin in Four Steps via Four-Component Coupling and Tetracyclization Steps. J. Am. Chem. Soc. 124, 11290-11291 (2002).

37. Riche, C. \& Pascard-Billy, C. Structure and stereochemistry of indole alkaloids. IV. Structure of andranginine. Acta Crystallogr., Sect. B B35, 666-669 (1979).

38. Kan-Fan, C. et al. Structure and biogenetic-type synthesis of andranginine: an indole alkaloid of a new type. J. Chem. Soc., Chem. Commun. 164-165 (1974).

39. Yanagisawa, A., Nomura, N. \& Yamamoto, H. Transition metal-catalyzed substitution reaction of allylic phosphates with Grignard reagents. Tetrahedron 50, 6017-6028 (1994).

40. Büchi, G., Kulsa, P., Ogasawara, K. \& Rosati, R. L. Syntheses of velbanamine and catharanthine. J. Am. Chem. Soc. 92, 999-1005 (1970).

41. Kutney, J. P. \& Bylsma, F. Synthesis of monomeric and dimeric vinca alkaloids. The total synthesis of isovelbanamine, velbanamine, cleavamine, $18 \beta$-carbomethoxycleavamine, and 
catharanthine. J Am. Chem. Soc. 92, 6090-6092 (1970).

42. Trost, B. M., Godleski, S. A. \& Belletire, J. L. Synthesis of ( \pm )-catharanthine via organopalladium chemistry. J. Org. Chem. 44, 2052-2054 (1979).

43. Atta-ur-Rahman, Beisler, J. A. \& Harley-Mason, J. The total syntheses of ( \pm )- $\alpha$ - and $\beta$-dihydrocleavamines, $( \pm)$-16-methoxycarbonyldihydrocleavamine, $( \pm)$-coronaridine, $( \pm$ )-dihydrocatharanthine, $( \pm)$-ibogamine, $( \pm)$-epi-ibogamine, and $( \pm)$-catharanthine. Tetrahedron 36, 1063-1070 (1980).

44. Marazano, C., Le, G. M. T., Fourrey, J. L. \& Das, B. C. An unequivocal synthesis of 1-benzyl-3-ethyl-1,6-dihydropyridine and its use for a biogenetically modeled synthesis of ( \pm )-catharanthine. J. Chem. Soc., Chem. Commun. 389-391 (1981).

45. Imanishi, T., Yagi, N., Shin, H. \& Hanaoka, M. 1,6-Dihydro-3(2H)-pyridinones. III. A formal synthesis of ( \pm )-catharanthine. Chem. Pharm. Bull. 30, 4052-4059 (1982).

46. Raucher, S. \& Bray, B. L. Total synthesis of ( \pm )-catharanthine. J. Org. Chem. 50, 3236-3237 (1985).

47. Raucher, S., Bray, B. L. \& Lawrence, R. F. Synthesis of ( \pm )-catharanthine, (+)-anhydrovinblastine, and (-)-anhydrovincovaline. J. Am. Chem. Soc. 109, 442-446 (1987).

48. Szántay, C., Bölcskei, H. \& Gács-Baitz, E. Synthesis of vinca alkaloids and related compounds XLVIII synthesis of (+)-catharanthine and ( \pm )-allocatharanthine. Tetrahedron 46, 1711-1732 (1990).

49. Reding, M. T., Kaburagi, Y., Tokuyama, H. \& Fukuyama, T. Synthesis of 2,3-disubstituted indoles by radical cyclization with hypophosphorous acid and its application to total synthesis of ( \pm )-catharanthine. Heterocycles 56, 313-330 (2002).

50. Moisan, L., Thuery, P., Nicolas, M., Doris, E. \& Rousseau, B. Formal synthesis of (+)-catharanthine. Angew. Chem. Int. Ed. 45, 5334-5336 (2006).

\section{Figure legends}

Figure 1 | Proposed biogenesis of indole alkaloids (5-7) and structure of ngouniensine (8). A hypothetical common intermediate, dehydrosecodine (4) could be biosynthesized through multistep enzymatic transformations starting from union of tryptamine (1) and secologanin (2). A pair of diene units in 4, a dihydropyridine (DHP) and a vinylindole, are thought to be installed through cleavage of two C-C bonds in the central core of an intermediate (3). Divergent [4+2] cyclizations of 4 would form the aspidosperma-type alkaloid tabersonine (5, path A) or the iboga-type catharanthine (6, path B). A distinct biosynthetic [4+2] cyclization is postulated for andranginine (7, path C), involving an intermediate bearing a cross-conjugated triene.

Figure 2 | Outline of a biogenetically inspired synthetic process to furnish alkaloidal scaffolds. a, 
Synthetic strategy for a divergent process featuring reactivity modulation of an achiral poly-unsaturated intermediate (14) through manipulation of the carbonyl group conjugated with the dihydropyridine (DHP) unit. Modular assembly of tryptamine (1) and building blocks (9, 10 and 12) followed by cyclization of ene-yne (13) would allow rapid and flexible access to the common intermediate (14). Three distinct modes of bioinspired [4+2] cyclizations could produce assortments of naturally occurring scaffolds (15-17). Distinct modes of annulations were devised through redox-activations of the DHP unit to furnish either the tetracyclic framework (18) of ngouniensine or the unnatural skeleton (19). b, Rapid formation of 1,6-DHP ring using $\mathrm{Cu}(\mathrm{I})$ catalysis. The optimum conditions for the 6-endo cyclization of ene-yne were explored using the model substrate (20). dppf, 1,1'-bis(diphenylphosphino)ferrocene.

Figure 3 | Unified synthetic process for divergent access to 23, 25, and 27 through distinct [4+2] cyclization reactions. Pictet-Spengler reaction of tryptamine (1) with 9 and subsequent ring expansion produced 22. Reduction followed by $N$-propargylation gave 11a. Assemblies of $\mathbf{1 1}$ with ethynylcarbonyl unit (12) could generate a zwitterionic intermediate effecting Hofmann elimination to afford ene-yne (13). Cu(I)-catalyzed cyclization of 13a followed by [4+2] cyclization allowed a streamlined synthesis of iboga-type scaffold 23. The aspidosperma-type scaffold (25b) was synthesized through regioselective hydrogenation of $\mathbf{1 4 b}$ and a different [4+2] cyclization. A distinct [4+2] cyclization was realized through conversion of $\mathbf{1 4 c}$ into $\mathbf{2 6 c}$ to produce andranginine-type scaffold (27). Boc, t-butoxycarbonyl; Teoc, 2-(trimethylsilyl)ethoxycarbonyl; Im, imidazole; dppf, 1,1'-bis(diphenylphosphino)ferrocene; DHP, dihydropyridine; TBAF, tetra-n-butylammonium fluoride; TIPSOTf, triisopropylsilyl trifluoromethanesulfonate.

Figure 4 | Redox-mediated activation of the DHP-vinylindole (14a) leading to tetracyclic scaffolds (28 and 29), which contain a DHP ring. The common intermediate (14a) generated from 11a was subjected to either two- or single-electron oxidation process to produce $\mathbf{2 8}$ and $\mathbf{2 9}$, respectively. Upon heating at $120^{\circ} \mathrm{C}$, ngouniensine-type scaffold (28) was obtained as the major product. The involvements of the retro-Diels-Alder reaction (23 $\rightarrow \mathbf{1 4 a}$ ), hydride shift followed by 7-endo-cyclization of a zwitterionic intermediate were postulated in the formation of $\mathbf{2 8}$. Single-electron oxidation of 14a provided an unnatural-type scaffold (29) presumably via 8-endo-radical cyclization. bpy, 2,2'-bipyridine; dppf, 1,1'-bis(diphenylphosphino)ferrocene.

Figure 5 | Total synthesis of three natural products. a, Site-selective reduction of methylester (25b) followed by oxidation of resulting primary alcohol and Wittig elongation gave $\mathbf{3 1}$.

Site-selective hydrogenation of $\mathbf{3 1}$ afforded ( \pm )-vincadifformine (32). b. Direct conversion of silylenol ether (27) to vinyl triflate (33) and subsequent palladium-catalyzed reduction furnished ( \pm )-andranginine (7). c, Hofmann elimination triggered by addition of 11a to 12c gave 34. After $\mathrm{Cu}(\mathrm{I})$-catalyzed formation of DHP ring, the critical [4+2] cyclization reaction proceeded with 
complete diastereocontrol in one-pot to produce 35. Removal of the chiral auxiliary, site-selective reduction of 36, and conversion into an allylic phosphate (37) followed by Fe-catalyzed cross-coupling with MeMgBr accomplished asymmetric total synthesis of (-)-catharanthine (6). DIBAL, diisobutylaluminium hydride; NaHMDS, sodium hexamethyldisilazide; Tf, trifluoromethanesulfonyl; dppf, 1,1'-bis(diphenylphosphino)ferrocene; acac, acetylacetonate.

\section{Acknowledgement}

The authors dedicate this manuscript to Masahiro Hirama on the occasion of his 65 th birthday. The authors thank Ms. Yuko Fujimura (Shionogi \& Co., Ltd.) and Mr. Takashi Matsumoto (Rigaku Corporation) for performing the X-ray analyses. This work was supported by JSPS KAKENHI (Grant No. 23310156 to H. Oguri) and in part by the Naito Foundation and the Science and Technology Research Partnership for Sustainable Development Program (SATREPS) of the Japan Science and Technology Agency (JST). A fellowship for H.M. from the JSPS is gratefully acknowledged.

\section{Contributions}

H.M. carried out the experimental work. H.M. and H. Oguri conceived the projects, analyzed the experimental results and wrote the manuscript. H. Oikawa discussed the results and provided oversight.

\section{Additional information}

Supplementary information and chemical compound information are available in the online version of the paper. Reprints and permission information is available online at http://www.nature.com/reprints. Correspondence and requests for materials should be addressed to $\mathrm{H}$. Oguri.

\section{Competing financial interests}

The authors declare no competing financial interests. 